\title{
Pilot study of feasibility and effect of anodal transcutaneous spinal direct current stimulation on chronic neuropathic pain after spinal cord injury
}

\author{
Young-Ah Choi ${ }^{1} \cdot$ Yale Kim ${ }^{1} \cdot$ Hyung-lk Shin ${ }^{1}$
}

Received: 9 October 2018 / Revised: 3 January 2019 / Accepted: 14 January 2019 / Published online: 30 January 2019

(c) International Spinal Cord Society 2019

\begin{abstract}
Study design A single-blind crossover study.

Objectives This study aimed to evaluate neuropathic pain in persons with spinal cord injury (SCI) after the application of transcutaneous spinal direct current stimulation (tsDCS).

Setting Outpatient Clinic of the Rehabilitation Department, Seoul National University Hospital.

Methods The effect of single sessions of both anodal and sham tsDCS (2 mA, $20 \mathrm{~min})$ on chronic neuropathic pain in ten volunteers with complete motor cervical SCI was assessed. The active electrode was placed over the spinal process of the tenth thoracic vertebra and the reference electrode, at the top of the head. Pre- to post-tsDCS intervention changes in pain intensity (numeric rating scale, NRS), patient global assessment, and present pain intensity (PPI) were assessed before and after the tsDCS session (immediately post stimulation, and at 1 and $2 \mathrm{~h}$ post stimulation).

Results All participants underwent the stimulation procedure without dropout. Our results showed no significant pre- to post-treatment difference in pain intensity between the active and sham tsDCS groups. Only in the sham tsDCS stimulation, NRS and PPI scores were reduced after the stimulation session. Furthermore, in the mixed effect model analysis, the response in the second period appeared to be more favorable.

Conclusion The results suggest that a single session of anodal tsDCS with the montage used in this study is feasible but does not have a significant analgesic effect in individuals with chronic cervical SCI.

Sponsorship The study was funded by Seoul National University Hospital (No. 0420160470) and Korea Workers' Compensation \& Welfare Service.
\end{abstract}

\section{Introduction}

Neuropathic pain is defined as 'pain caused by a lesion or disease of the somatosensory nervous system' by the International Association for the Study of Pain [1]. Neuropathic pain after spinal cord injury (SCI) is one of the most difficult complications to treat, and the prevalence of the symptom has been reported to be experienced by $\sim 34 \%$ of

Supplementary information The online version of this article (https:// doi.org/10.1038/s41393-019-0244-x) contains supplementary material, which is available to authorized users.

Hyung-Ik Shin

hyungik1@snu.ac.kr

1 Department of Rehabilitation Medicine, College of Medicine, Seoul National University, Seoul, Republic of Korea persons with SCI [2]. The most common treatment for neuropathic pain is pharmacotherapy, including antidepressants, anticonvulsants, local anesthetics, and narcotics $[3,4]$. Unfortunately, oral pharmaceutical intervention commonly results in a reduction of only $20-30 \%$ in pain intensity [5], and Siddall et al. reported that only $4-6 \%$ of persons with SCI experienced relief from neuropathic pain with medication [6]. Recent neuroimaging and neurophysiological studies have shown that the neural mechanism causing chronic pain results mainly from maladaptive plastic changes [7].

Several non-pharmacologic treatments such as cranial electrotherapy stimulation, transcutaneous electrical nerve stimulation, repetitive transcranial magnetic stimulation, and transcranial direct current stimulation (tDCS) are emerging as alternatives to treat chronic neuropathic pain in persons with SCI. These methods have the advantage of minimal side effects [8]. According 
to a recent meta-analysis, the only non-pharmacologic treatment that reduced chronic pain in people with SCI was tDCS [9]. tDCS over the primary motor cortex exerts long-lasting modulatory effects on cortical functioning by developing a constant electrical field $[10,11]$ and modulating emotional and cognitive components of pain and normalizing excessive attention to pain and pain-related information [12]. Despite these promising findings, mixed results have been reported, especially regarding the duration of the effect [13-15].

The well-established effectiveness of invasive spinal cord stimulation for chronic pain suggests that direct current stimulation could also be applied to the spinal cord noninvasively (tsDCS) [16-19]. The tsDCS modeling study conducted by Parazzini et al. showed that the current density and electric field tended to be primarily directed longitudinally along the spinal cord [20]. Several neurophysiological studies have shown modulating effects of tsDCS on nociceptive signal processing in healthy people. Cogiamanian et al. first showed that anodal tsDCS reduces cervical vertebra-brain stem somatosensory evoked potential amplitude for up to 20 min [21]. Truini et al. demonstrated that anodal spinal tsDCS reduces laser-evoked potential amplitude, which involves pain signals transmitted to the brain via the spinothalamic tract after activation of A delta fibers [22]. Regarding spinal cord segmental circuits, Cogiamanian et al. showed that anodal spinal tsDCS induces longlasting depression of the flexion reflex late response [23]. This suggests that anodal spinal tsDCS may reduce painful reflexes and may be associated with analgesic effects. Furthermore, anodal tsDCS can suppress the pain sensitivity associated with nociceptive mechanical stimuli [24], and anodal tsDCS can modulate temporal summation of pain based on depression of the transitory facilitation of the activity of wide dynamic range neurons [25]. In brief, tsDCS modulates neuronal activity in lemniscal, spinothalamic, and segmental spinal circuits, suggesting glutamatergic, GABAergic, and glycinergic system involvement, and eventual effects on spinal plasticity [26, 27]. However, in contrast to the brain stimulation approach, few clinical studies have assessed the effects of tsDCS in individuals with SCI.

Since a previous modeling study used a realistic human model rather than human participants, the current study was conducted to evaluate the feasibility of tsDCS in actual clinical practice. Additionally, we tried to evaluate the effectiveness of tsDCS using a montage with an active electrode attached to the thoracic vertebra and a reference electrode attached to the head vertex for relieving neuropathic pain in persons with SCI.

\section{Methods}

\section{Participants}

Participants were prospectively recruited from the Outpatient Clinic of the Rehabilitation Department, Seoul National University Hospital, from October 2016 to April 2017. This pilot study was based on a convenient sample of ten participants who expressed willingness to participate. The inclusion criteria were as follows: (1) cervical complete motor SCI, at least 6 months after SCI; (2) 20 years $\leq$ age $<90$ years; (3) Leeds assessment of neuropathic symptoms and signs (LANSS) score $\geq 12$ (LANSS estimates the probability of neuropathic mechanisms contributing to the chronic pain experience in a given patient; if score $<12$, then neuropathic mechanisms are unlikely to contribute to the patient's pain and if score $\geq 12$, then neuropathic mechanisms are likely to contribute to the patient's pain) [28-31]; (4) continuous chronic pain for at least the three preceding months and a score $\geq 4(0 \mathrm{~cm}=$ 'no pain,' and $10 \mathrm{~cm}=$ 'worst possible pain') on a visual analog scale for pain perception at the baseline/start of the treatment; and (5) refractoriness to drugs for pain relief, such as antidepressants, antiepileptics, and/or opioids (pain resistant to at least two of these drugs supplied in adequate doses for 6 months).

The exclusion criteria were: (1) any clinically significant or unstable medical or progressive neurologic disorder; (2) contraindication for electrical stimulation, such as a pacemaker implant; (3) childbearing age or pregnancy; (4) significant cognitive deficit; (5) syringomyelia; (6) neuropsychiatric comorbidity; (7) depressive disorder (as indicated by a score $>10$ on the Beck Depression Inventory) [32]; (8) history of substance abuse; (9) skin defect where the electrodes would be placed; and (10) progressive neurological disease or other secondary conditions that could impact neuropathic pain. All participants read and provided signed informed consent. The study was conducted in accordance with the Declaration of Helsinki and was approved by the Ethics Committee of Seoul National University Hospital (identifier number: 1604-007-752). Since the application of tsDCS for neuropathic pain in persons with SCI has not yet been certified, our clinical experiment was approved and registered by the Ministry of Food and Drug Safety (registration number: 864) in South Korea. The registration number at http://www.clinicaltrials. gov was NCT02863315.

\section{Study design and experimental procedures}

A single-blinded, randomized, crossover layout design was used. All participants participated in each single session of 
anodal tsDCS and sham treatment period. The two conditions were conducted in a random order using block randomization, which was generated using a computerized random sequence allocation by the study principal investigator, who was not involved in data collection or participant orientation and supervision. Baseline and post-intervention assessments were conducted by a physiotherapist. Pain and other pain-related variables were assessed immediately before (baseline) and immediately (T0), $1 \mathrm{~h}$ (T1), and $2 \mathrm{~h}$ (T2) after the stimulation offset. The washout period was two weeks between sessions to avoid any carry-over effect and the participants were blinded to the conditioning stimulation. Participants were requested not to cease or change medications during the trial and to continue their routine analgesic medication regimen throughout the duration of the study. At each time point, the participants were checked for their actual medication use.

\section{Transcutaneous spinal DC stimulation (tsDCS)}

Direct current stimulation ( $2 \mathrm{~mA}, 20 \mathrm{~min}$ ) was delivered by a constant current electrical stimulator, DC-Stimulator Plus (NeuroConn GmbH, Ilmenaus, Germany) [33]. The cathodal (reference) electrode was positioned over the head vertex (in the $\mathrm{Cz}$ location according to the 10-20 EEG system) and the anodal (active) electrode was positioned over the thoracic spinal cord (over the spinous process of the tenth thoracic vertebra) (Fig. 1). Both electrodes were 6 $\mathrm{mm}$ thick, square $\left(35 \mathrm{~cm}^{2}\right)$ pieces of saline-soaked synthetic sponge. Elastic straps were applied to ensure that the electrodes were accurately located [34]. For sham tsDCS (placebo), the same procedure was used, but the simulator was turned off after $30 \mathrm{~s}$ to simulate an initial tingling sensation like that felt at the beginning of active stimulation. Stimulation was applied while the participant was in seated in a wheelchair.

\section{Measures}

The subjective perception of pain before and after stimulation, as a primary outcome, was estimated by a numeric rating scale (NRS) for neuropathic pain on a scale of 0 to 10 , with 0 being no pain and 10 being the worst pain imaginable [35]. We used the patient global assessment (PGA) and present pain intensity (PPI) as secondary outcome measures. The PGA is a single-item rating by participants of their improvement after treatment on a 7-point scale that ranges from "very much improved" to "very much worse," with "no change" as the midpoint [36]. The overall PPI, a major component of the McGill Pain Questionnaire, provides data on intensity. It is recorded as a number from 1 to 5 (1, mild; 2, discomforting; 3 ,

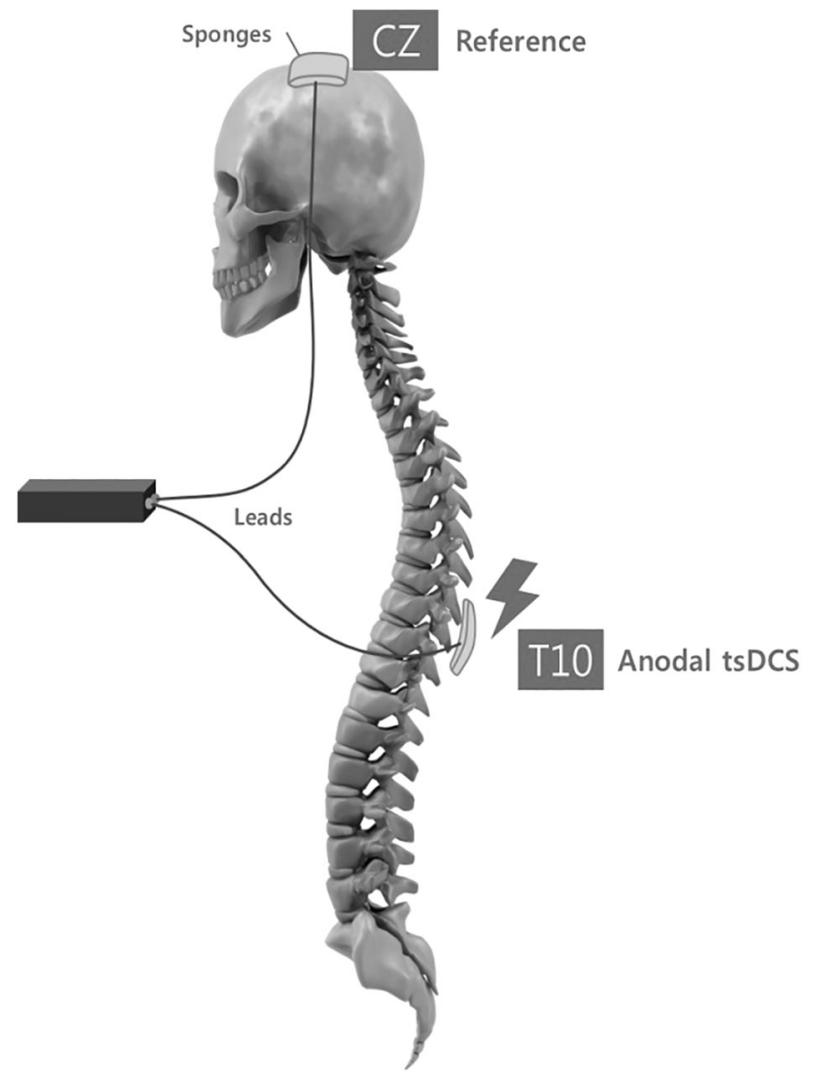

Fig. 1 Schematic of electrode placement for tsDCS. The reference electrode was positioned over the head vertex (in the $\mathrm{Cz}$ location according to the 10-20 EEG system) and the anodal electrode was positioned over the spinous process of the tenth thoracic vertebra

distressing; 4, horrible; 5, excruciating) [37]. Finally, participants were asked to report any adverse events that occurred immediately after treatment and again at 24- and $48 \mathrm{~h}$ after each of the stimulation sessions. Physicians conducted careful observations throughout the study, with vital sign monitoring.

\section{Data and statistical analysis}

The demographic and outcome variables are expressed as means and standard deviations (SD). We compared the primary and secondary outcome measures obtained during the baseline period between participants assigned to the two treatment orders (i.e., active-sham versus sham-active). The Wilcoxon signed-rank test was used to assess significant differences between active and sham tsDCS treatment in NRS, PGA, and PPI at specific time-points (baseline, immediately [T0], $1 \mathrm{~h}$ [T1], and $2 \mathrm{~h}$ [T2] after the stimulation offset). Because of the small sample size, the homogeneity of the carry-over effect in each of the two sequences was not evaluated. It was assumed that there was no carryover effect owing to the two-week washout periods. 
Fig. 2 CONSORT flow diagram. CONSORT flow diagram showing ten eligible participants through each stage of the singleblinded, randomized, crossover controlled trial (enrollment, intervention allocation, followup, and data analysis)

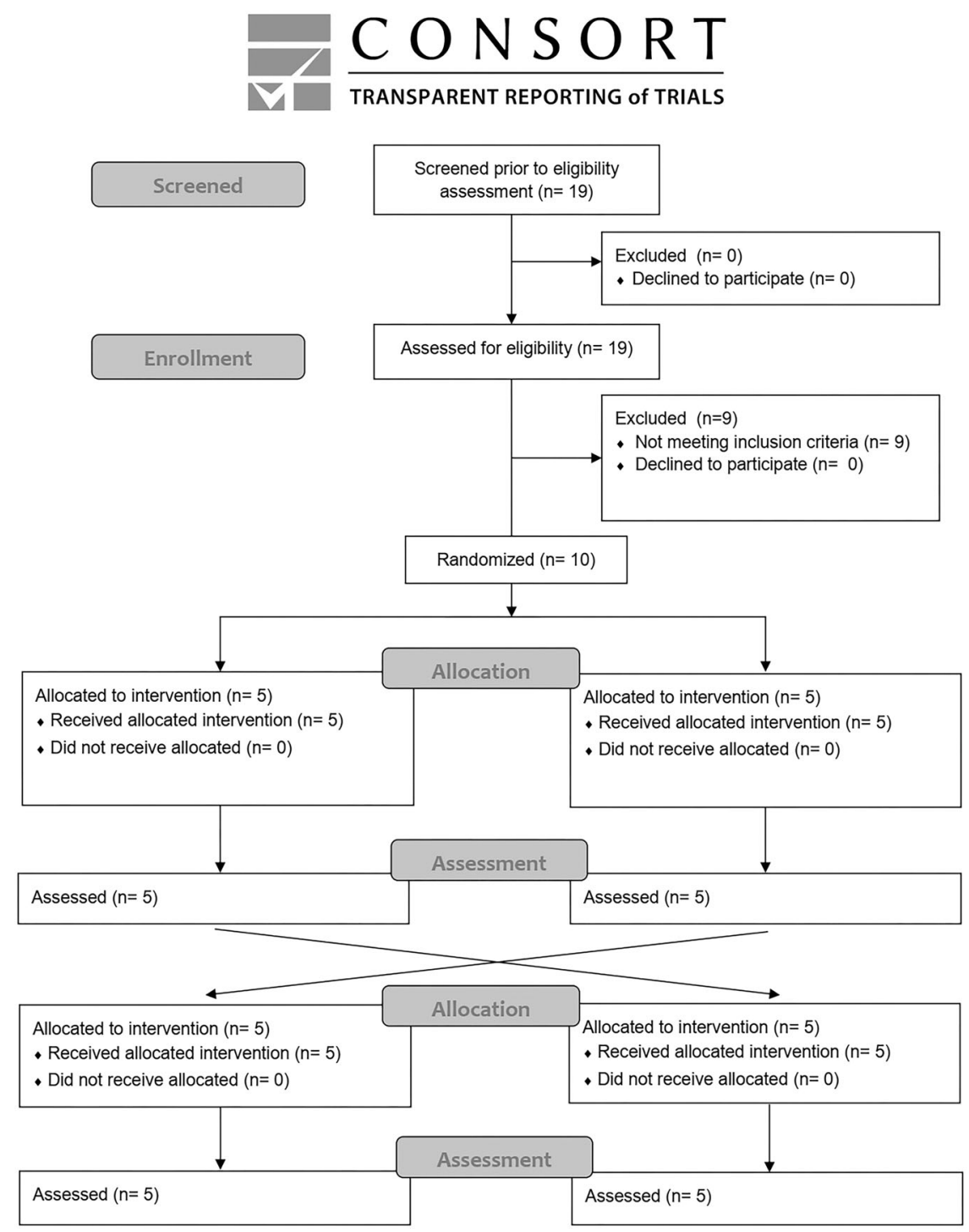

In addition, we used a non-parametric mixed effects model with fixed effects of treatment, treatment period, time point of assessment, the interaction between treatment and time, the interaction between period and time, and carry-over effect, and a random effect of participants (nested in sequence). The correlation matrix between the measured data of participants at each assessment time point was used as the first order autoregressive structure. If carry-over effects and interaction effects were non-significant at a significance level of 0.05 , the model was rebuilt without those effects. The model was first fitted with the pain intensity data. All participants were included, and all pairwise comparisons were made with the estimated contrast from this model. Secondary outcome measures were also analyzed in the same way as the primary outcome. We analyzed data using the intent-to-treat method, and $p$ values $<0.05$ were considered statistically significant.
Analyses were completed using SPSS Statistics (SPSS Inc, 233 S Wacker Dr, 11th Fl, Chicago, IL 60606).

\section{Results}

\section{Baseline demographic data}

Nineteen participants with traumatic SCI experiencing neuropathic pain were screened. The ten eligible participants included seven men and three women (Fig. 2). The demographic and clinical characteristics at baseline are summarized in Table 1. When explicitly asked, none of the participants could tell whether the stimulation was active or sham. Similar to perceived pain intensity, we found that pain unpleasantness was not affected by the active or sham 

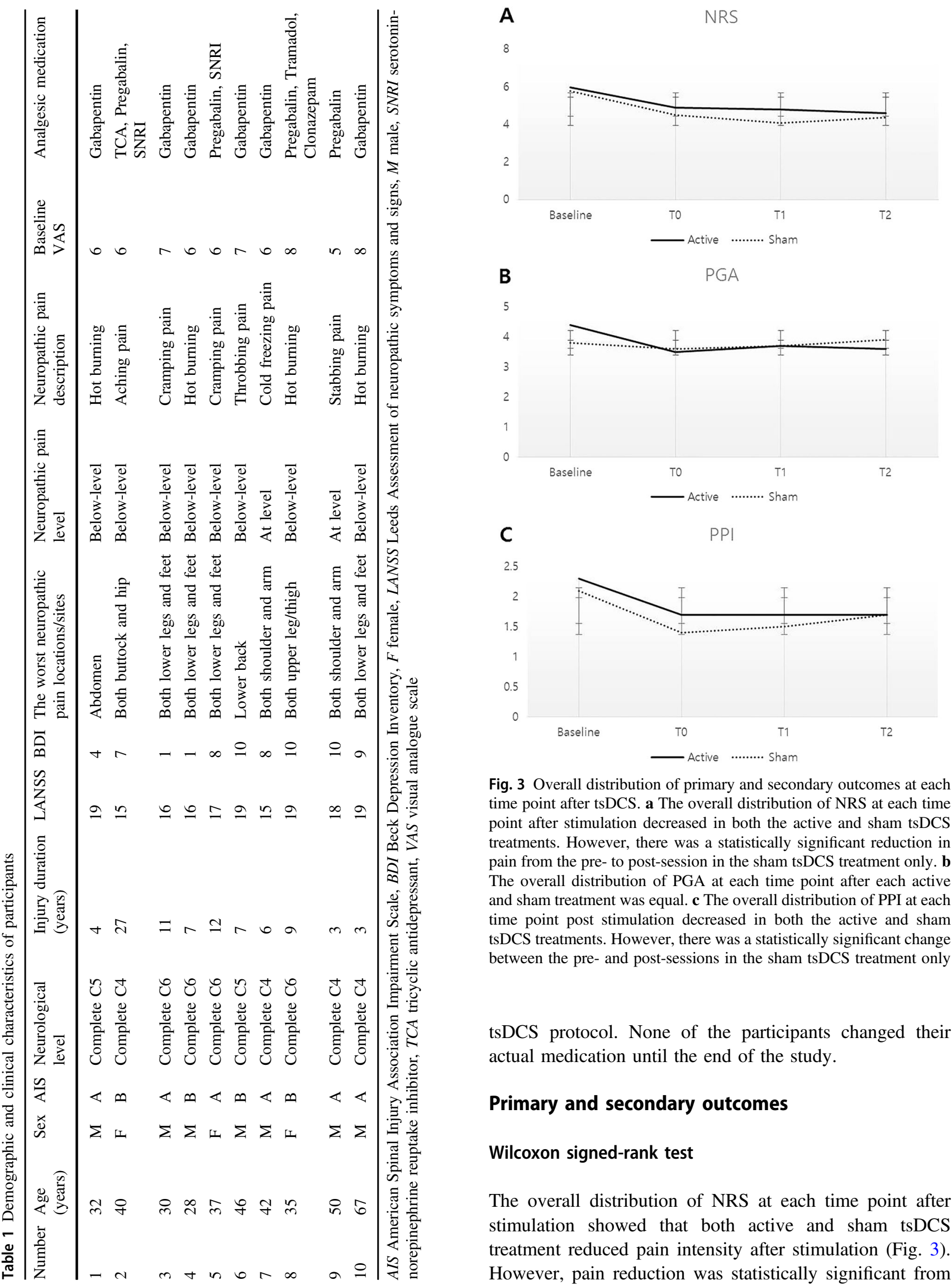

Fig. 3 Overall distribution of primary and secondary outcomes at each time point after tsDCS. a The overall distribution of NRS at each time point after stimulation decreased in both the active and sham tsDCS treatments. However, there was a statistically significant reduction in pain from the pre- to post-session in the sham tsDCS treatment only. b The overall distribution of PGA at each time point after each active and sham treatment was equal. $\mathbf{c}$ The overall distribution of PPI at each time point post stimulation decreased in both the active and sham tsDCS treatments. However, there was a statistically significant change between the pre- and post-sessions in the sham tsDCS treatment only

tsDCS protocol. None of the participants changed their actual medication until the end of the study.

\section{Primary and secondary outcomes}

\section{Wilcoxon signed-rank test}

The overall distribution of NRS at each time point after stimulation showed that both active and sham tsDCS treatment reduced pain intensity after stimulation (Fig. 3). However, pain reduction was statistically significant from 
Table 2 Wilcoxon signed-rank test for active and sham tsDCS

\begin{tabular}{|c|c|c|c|c|c|c|c|}
\hline \multirow[t]{2}{*}{ Parameter } & \multirow[t]{2}{*}{ Time point } & \multicolumn{4}{|c|}{ Sham $(n=10)$} & \multicolumn{2}{|c|}{ Active $(n=10)$} \\
\hline & & Mean \pm SD & $p$-value ${ }^{2}$ & $p$-value ${ }^{3}$ & $p$-value ${ }^{4}$ & Mean \pm SD & $p$-value ${ }^{5}$ \\
\hline \multirow[t]{5}{*}{ NRS } & Baseline & $5.8 \pm 1.32$ & & & & $6.0 \pm 1.33$ & \\
\hline & T0 & $4.5 \pm 1.95$ & $0.0313 *$ & & & $4.9 \pm 2.08$ & 0.8125 \\
\hline & $\mathrm{T} 1$ & $4.1 \pm 2.23$ & $0.0126^{*}$ & 0.4373 & & $4.8 \pm 1.81$ & 0.5469 \\
\hline & $\mathrm{T} 2$ & $4.4 \pm 2.41$ & 0.1897 & 0.6882 & 0.1950 & $4.6 \pm 2.01$ & 1.0000 \\
\hline & $p$-value ${ }^{1}$ & $0.0102 *$ & & & & 0.3346 & \\
\hline \multirow[t]{5}{*}{ PGA } & Baseline & $3.8 \pm 0.79$ & & & & $4.4 \pm 0.84$ & \\
\hline & T0 & $3.6 \pm 0.97$ & & & & $3.5 \pm 0.97$ & 0.1406 \\
\hline & $\mathrm{T} 1$ & $3.7 \pm 0.82$ & & & & $3.7 \pm 0.67$ & 0.2734 \\
\hline & $\mathrm{T} 2$ & $3.9 \pm 1.10$ & & & & $3.6 \pm 0.84$ & 0.0859 \\
\hline & $p$-value ${ }^{1}$ & 0.9135 & & & & 0.0779 & \\
\hline \multirow[t]{5}{*}{ PPI } & Baseline & $2.1 \pm 0.74$ & & & & $2.3 \pm 0.82$ & \\
\hline & T0 & $1.4 \pm 0.70$ & $0.0124 *$ & & & $1.7 \pm 0.67$ & 1.0000 \\
\hline & $\mathrm{T} 1$ & $1.5 \pm 0.71$ & $0.0135^{*}$ & 0.3173 & & $1.7 \pm 0.82$ & 1.0000 \\
\hline & $\mathrm{T} 2$ & $1.7 \pm 0.82$ & 0.1709 & 0.0841 & 0.1874 & $1.7 \pm 0.82$ & 0.8750 \\
\hline & $p$-value ${ }^{1}$ & $0.0452 *$ & & & & 0.1442 & \\
\hline
\end{tabular}

NRS numeric rating scale, $P G A$ patient global assessment, $P P I$

(1) Non-parametric test results for distribution homogeneity at each time point

$(2,3,4)$ Post hoc analyses of the time factor for the sham condition

(5) Wilcoxon signed-rank test for pre- to each post-treatment difference between the sham and active tsDCS groups

$* p<0.05$

the pre- to post-session in the sham tsDCS group only ( $p=$ 0.0102). We performed post hoc analyses of the time factor for the sham condition. There was a significant change in pain intensity immediately after stimulation (T0), and at $1 \mathrm{~h}$ post treatment (T1) compared to baseline.

There was no effect of tsDCS on PGA scores for either the active or sham tsDCS treatments at each time point post treatment, as assessed using a non-parametric repeated measurements test $(p=0.0779, p=0.9135$, respectively).

The overall distribution of PPI at each time point post stimulation showed that both the active and sham tsDCS treatments decreased PPI scores. However, the PPI distributions at baseline, immediately (T0), $1 \mathrm{~h}$ (T1), and $2 \mathrm{~h}$ (T2) after stimulation offset were significantly different in the sham tsDCS group only $(p=0.0452)$. Post hoc analyses of the time factor for the sham condition indicated that there was a significant change in pain intensity compared to baseline immediately after stimulation (T0) and at $1 \mathrm{~h}$ post treatment (T1).

Comparing the pre- to post-treatment difference between the active and sham tsDCS groups, the Wilcoxon signedrank test for each group showed no significant decrease in pain intensity (NRS) for the active tsDCS group. Furthermore, there was no significant difference between the active and sham tsDCS groups with regard to both the PGA and PPI scores (Table 2).

\section{Mixed effects model}

The interactions between treatment and assessment timepoints, and treatment period and assessment time-points, were not significant; hence, the model was reconstructed excluding those carry-over effects. The mean NRS pain scores for the treatments did not significantly differ as a function of treatment. The mean NRS of the second period was 1 point lower than that of the first period. At each assessment time point, the mean NRS showed a tendency to be 1 point lower compared to the baseline NRS. The mean PGA also did not differ as a function of treatment. The mean PGA of the second period was 0.5 points lower than that of the first period. Compared to the baseline PGA, the mean PGA at each assessment time point showed a tendency to be 0.5 points lower. Finally, there was no significant difference in the mean PPI as a function of treatment. The mean PPI of the second period was 0.6 points lower than that of the first period, and the mean PGA at each assessment time point showed a tendency to be 0.5 points lower compared to the baseline PGA (Table 3 ).

\section{Adverse events provide sufficient detail to allow the work to be reproduced}

All participants tolerated the stimulation procedures well without dropout (see supplementary material). Although 
Table 3 Mixed effects model for active and sham tsDCS

\begin{tabular}{|c|c|c|c|c|c|}
\hline Parameter & & & LSMean $[95 \% \mathrm{CI}]$ & LSMean difference & $p$-value \\
\hline \multirow[t]{9}{*}{ NRS } & \multirow[t]{2}{*}{ Treatment } & Sham & $4.9[3.89,5.87]$ & & 0.9908 \\
\hline & & Active & $4.9[3.89,5.87]$ & $0.0[-0.75,0.74]$ & \\
\hline & \multirow[t]{2}{*}{ Period } & Period 1 & $5.4[4.37,6.35]$ & & $0.0132 *$ \\
\hline & & Period 2 & $4.4[3.42,5.39]$ & $-1.0[-1.70,-0.21]$ & \\
\hline & \multirow[t]{5}{*}{ Time } & & & & $0.0004 *$ \\
\hline & & Baseline & $5.9[4.88,6.92]$ & & \\
\hline & & T0 & $4.7[3.66,5.69]$ & $-1.2[-2.01,-0.44]$ & $0.0027 *$ \\
\hline & & $\mathrm{T} 1$ & $4.5[3.43,5.47]$ & $-1.5[-2.21,-0.69]$ & $0.0003^{*}$ \\
\hline & & $\mathrm{T} 2$ & $4.5[3.48,5.52]$ & $-1.4[-2.07,-0.73]$ & $<0.0001 *$ \\
\hline \multirow[t]{9}{*}{ PGA } & \multirow[t]{2}{*}{ Treatment } & Sham & $3.8[3.45,4.23]$ & & 0.5276 \\
\hline & & Active & $3.7[3.32,4.10]$ & $-0.1[-0.57,0.30]$ & \\
\hline & \multirow[t]{2}{*}{ Period } & Period 1 & $4.0[3.65,4.43]$ & & $0.0174 *$ \\
\hline & & Period 2 & $3.5[3.12,3.90]$ & $-0.5[-0.96,-0.10]$ & \\
\hline & \multirow[t]{5}{*}{ Time } & & & & 0.1375 \\
\hline & & Baseline & $4.1[3.67,4.53]$ & & \\
\hline & & T0 & $3.6[3.12,3.98]$ & $-0.6[-1.03,-0.07]$ & $0.0027^{*}$ \\
\hline & & $\mathrm{T} 1$ & $3.7[3.27,4.13]$ & $-0.4[-0.87,0.07]$ & $0.0003^{*}$ \\
\hline & & $\mathrm{T} 2$ & $3.8[3.32,4.18]$ & $-0.4[-0.78,0.08]$ & $<0.0001 *$ \\
\hline \multirow[t]{9}{*}{ PPI } & \multirow[t]{2}{*}{ Treatment } & Sham & $1.8[1.44,2.15]$ & & 0.6845 \\
\hline & & Active & $1.7[1.38,2.08]$ & $-0.1[-0.38,0.25]$ & \\
\hline & \multirow[t]{2}{*}{ Period } & Period 1 & $2.1[1.72,2.43]$ & & $0.0002 *$ \\
\hline & & Period 2 & $1.4[1.10,1.80]$ & $-0.6[-0.94,-0.31]$ & \\
\hline & \multirow[t]{5}{*}{ Time } & & & & $0.0009^{*}$ \\
\hline & & Baseline & $2.2[1.83,2.57]$ & & \\
\hline & & T0 & $1.6[1.18,1.92]$ & $-0.7[-0.99,-0.31]$ & $0.0003 *$ \\
\hline & & $\mathrm{T} 1$ & $1.6[1.23,1.97]$ & $-0.6[-0.93,-0.27]$ & $0.0006^{*}$ \\
\hline & & $\mathrm{T} 2$ & $1.7[1.33,2.07]$ & $-0.5[-0.80,-0.20]$ & $0.0013^{*}$ \\
\hline
\end{tabular}

LSMean Least-squares means, NRS numeric rating scale, PGA patient global assessment, PPI present pain intensity

$* p<0.05$

most participants felt tingling at the anodal or cathodal site, the tingling sensation was transient and tolerated.

\section{Discussion}

Our results demonstrated that the application of tsDCS for neuropathic pain after SCI is feasible. All participants tolerated the stimulation procedure without dropout. However, at least in this participant group, there was no significant pre- to post-treatment difference in pain intensity (NRS), and PGA and PPI scores between active and sham treatment of tsDCS. Many previous studies have clinically applied tDCS to neuropathic pain after SCI; however, only the electrophysiologic effects of tsDCS on nociceptive signaling pathways in healthy participants were assessed. To the best of our knowledge, this preliminary study is the first pilot study to apply tsDCS clinically for neuropathic pain after SCI.
Reduction of NRS and PPI from the pre- to post-session was statistically significant in the sham tsDCS group only. Furthermore, although a carry-over effect was not observed in the mixed effect model analysis, the response in the second period appears to be more favorable. These results might represent adaptation to spinal direct current stimulation. The NRS, PGA, and PPI tended to slightly improve by $1,0.5$, and 0.5 points, respectively, after stimulation. However, the difference was $<2$ points for the NRS, which is regarded as the minimum change reflecting clinically important differences in pain outcome measures [38, 39]; thus, it is difficult to regard this as a clinically meaningful reduction in pain.

Recent neurophysiological evidence has shown suppressive effects of anodal tsDCS on nociceptive signal processing. In addition to the modulating effects at an electrophysiological level, anodal tsDCS significantly decreased subjective pain reports to painful mechanical stimuli in humans and induced early and long-lasting 
depression of the transitory facilitation of wide dynamic range neuronal activity $[24,25]$. Nonetheless, we failed to detect significant differences between anodal and sham tsDCS.

In the present study, a single 20-min session might not have been sufficient to reduce neuropathic pain in persons with SCI. Boggio et al. demonstrated that weekly sessions of tDCS were no more effective than a single tDCS session in individuals with stroke [40]. Furthermore, in the study by Ngernyam et al., a single session of tDCS was effective for neuropathic pain in individuals with SCI. With regard to brain stimulation [41], the pain modulation effect of tDCS might work early in the treatment phase, and prolonged stimulation may decrease responsiveness in some individuals. However, we speculate that for the spinal mechanism, unlike brain stimulation, consecutive stimulations, as opposed to a single stimulation, are required to show efficacy. Further research is needed to determine the pain reducing effect of consecutive sessions of tsDCS. Schweizer et al. induced hyperalgesia associated with transient receptor potential cation channel subfamily $\mathrm{V}$ member 1 (TRPV1)-positive $\mathrm{C}$ and $\mathrm{A}$ fibers using the high frequency stimulation model for long-term potentiation-like pain. Neither anodal nor cathodal tsDCS had any effects on pain perception associated with long-term potentiation-like pain in healthy participants after sensitization [42]. Neuropathic pain in persons with chronic SCI in this study might represent a similar condition to the sensitized state, and application of anodal tsDCS has no effect on pain relief, even in our study. It could be considered that the modulating effect of anodal tsDCS on nociceptive afferent fibers might be modality-specific, and so it is necessary to evaluate the effectiveness of tsDCS by subdividing neuropathic pain into detailed phenotypes in further studies. Considering the characteristics of the participants group, the mean $( \pm \mathrm{SD})$ injury duration of our group was relatively long $(8.9 \pm 6.71$ years). Wrigley et al. proposed that the long injury duration of their participants $(21.3 \pm 13.8$ years $)$ may explain why tDCS did not work [13]. They hypothesized that a change in central pain transmission circuits after SCI becomes consolidated over a long period of time; therefore, tDCS could not modulate the central pain-related system in persons with chronic SCI, significantly reducing the analgesic properties. However, Yoon et al. suggested that the long injury period would not contribute to the lack of tDCS effectiveness [12].

In terms of the technical features of tsDCS, stimulation intensity and duration were kept relatively similar among the various studies, with intensities between 2 and $2.5 \mathrm{~mA}$ and duration between 15 and $20 \mathrm{~min}$ [33]. However, the position of the reference electrode can potentially influence the induced current density distribution. Parazzini and colleagues first estimated the current density spatial distributions generated by tsDCS with various human models [20]. Three electrode montages were applied. All anode electrodes were placed on the 10th thoracic vertebra. The reference electrode was different for each montage. Montage A was attached to the right arm, Montage B was attached to the umblicus, and Montage $\mathrm{C}$ was attached to $\mathrm{Cz}$ on the 10-20 EEG system. In all realistic human models, the magnitude of the current density was largest in the cervical region when Montage $\mathrm{C}$ was applied. Furthermore, Montage $\mathrm{C}$ generated field amplitudes at the thoracic level that were remarkably higher (around double on average) than Montage A and Montage B. Based on the tsDCS modeling study by Parazzini et al., the current density distribution with a reference electrode over $\mathrm{Cz}$ could double its physiological effects by the site of action at both the spinal and supraspinal bulbar levels [20]. Assuming the spinal cord below the level of the injury is a disorganized and disconnected neural circuit owing to the lack of supraspinal control, the montage with the reference electrode on $\mathrm{Cz}$ was applied to maximize the spinal plastic effects of tsDCS in this study.

This study has some limitations. First, only a small number of participants $(n=10)$ met the inclusion criteria. A larger sample size may have detected an effect that was missed in this study. Since this pilot study aimed to evaluate the feasibility of treatment with tsDCS in persons experiencing chronic neuropathic pain after SCI, a significant analgesic effect could possibly be observed if participant numbers were increased. Second, this randomized study was single- rather than double-blinded, and so experimenter bias cannot be completely excluded. However, we tried to minimize bias by using a physiotherapist who was blinded to group assignment to record the measurements. Finally, the short follow-up of up to $4 \mathrm{~h}$ could be the reason for not observing any long-term pain reduction effect.

Although tsDCS may represent a promising non-invasive neuromodulatory tool to reduce chronic neuropathic pain by targeting the pain-maladaptive plasticity that develops after SCI, our data showed no beneficial effects for a single 20 min session of tDCS on neuropathic pain intensity in persons with SCI. Further studies are needed with changes such as targeting the early phase after SCI and applying consecutive stimulation protocols of tsDCS.

\section{Data archiving}

All data generated or analyzed during this study are included in this published article and its supplementary material file.

Acknowledgements We thank the Division of Medical Statistics, Medical Research Collaborating Center (MRCC) at Seoul National University Hospital for their support for statistical analyses. 
Funding This study was funded by Seoul National University Hospital (No. 0420160470) and Korea Workers' Compensation \& Welfare Service.

Author contributions YAC was responsible for designing the study, collecting data, extracting and analyzing data, interpreting results, and writing the report. YK was responsible for collecting data. HIS was responsible for designing the study, collecting data, interpreting the results, and writing the report. He provided feedback on the report and approved the final version.

\section{Compliance with ethical standards}

Conflict of interest The authors declare that they have no conflict of interest.

Statement of ethics The study was conducted in accordance with the Declaration of Helsinki and was approved by the Ethics Committee of Seoul National University Hospital (identifier number: 1604-007-752). We certify that all applicable institutional and governmental regulations concerning the ethical use of human volunteers were followed during the course of this research.

Publisher's note: Springer Nature remains neutral with regard to jurisdictional claims in published maps and institutional affiliations.

\section{References}

1. Haanpää M, Attal N, Backonja $M$, Baron $R$, Bennett $M$, Bouhassira D, et al. NeuPSIG guidelines on neuropathic pain assessment. Pain. 2011;152:14-27.

2. Soler MD, Sauri-Ruiz J, Curcoll-Gallemi ML, Benito-Penalva J, Opisso-Salleras E, Chamarro-Lusar A, et al. Characteristics of chronic neuropathic pain and their relationship with psychological well-being in spinal cord injury patients. Rev Neurol. 2007;44:3-9.

3. Mehta S, McIntyre A, Janzen S, Loh E, Teasell R. Systematic review of pharmacologic treatments of pain after spinal cord injury: an update. Arch Phys Med Rehabil. 2016;97:1381-91.

4. Dworkin RH, O'Connor AB, Backonja M, Farrar JT, Finnerup NB, Jensen TS, et al. Pharmacologic management of neuropathic pain: evidence-based recommendations. Pain. 2007;132:237-51.

5. Baastrup C, Finnerup NB. Pharmacological management of neuropathic pain following spinal cord injury. CNS Drugs. 2008;22:455-75.

6. Siddall PJ, McClelland JM, Rutkowski SB, Cousins MJ. A longitudinal study of the prevalence and characteristics of pain in the first 5 years following spinal cord injury. Pain. 2003;103:249-57.

7. Wagner $T$, Valero-Cabre A, Pascual-Leone A. Noninvasive human brain stimulation. Annu Rev Biomed Eng. 2007;9:527-65.

8. Moreno-Duarte I, Morse LR, Alam M, Bikson M, Zafonte R, Fregni F. Targeted therapies using electrical and magnetic neural stimulation for the treatment of chronic pain in spinal cord injury. Neuroimage. 2014;85:1003-13.

9. Boldt I, Eriks-Hoogland I, Brinkhof MW, de Bie R, Joggi D, von Elm E. Non-pharmacological interventions for chronic pain in people with spinal cord injury. Cochrane Database Syst Rev. 2014;11:CD009177.

10. Nitsche MA, Paulus W. Sustained excitability elevations induced by transcranial DC motor cortex stimulation in humans. Neurology. 2001;57:1899-901.

11. Nitsche MA, Liebetanz D, Lang N, Antal A, Tergau F, Paulus W. Safety criteria for transcranial direct current stimulation (tDCS) in humans. Clin Neurophysiol. 2003;114:2220-2.
12. Yoon EJ, Kim YK, Kim H-R, Kim SE, Lee Y, Shin HI. Transcranial direct current stimulation to lessen neuropathic pain after spinal cord injury: a mechanistic PET study. Neurorehabil Neural Repair. 2014;28:250-9.

13. Wrigley PJ, Gustin SM, McIndoe LN, Chakiath RJ, Henderson LA, Siddall PJ. Longstanding neuropathic pain after spinal cord injury is refractory to transcranial direct current stimulation: a randomized controlled trial. Pain. 2013;154:2178-84.

14. Fregni F, Boggio PS, Lima MC, Ferreira MJ, Wagner T, Rigonatti SP, et al. A sham-controlled, phase II trial of transcranial direct current stimulation for the treatment of central pain in traumatic spinal cord injury. Pain. 2006;122:197-209.

15. Soler MD, Kumru H, Pelayo R, Vidal J, Tormos JM, Fregni F, et al. Effectiveness of transcranial direct current stimulation and visual illusion on neuropathic pain in spinal cord injury. Brain. 2010;133:2565-77.

16. Cogiamanian F, Ardolino G, Vergari M, Ferrucci R, Ciocca M, Scelzo E, et al. Transcutaneous spinal direct current stimulation. Front Psychiatry. 2012;3:63.

17. Lamy JC, Boakye M. BDNF Val66Met polymorphism alters spinal DC stimulation-induced plasticity in humans. J Neurophysiol. 2013;110:109-16.

18. Bolognini N, Pascual-Leone A, Fregni F. Using non-invasive brain stimulation to augment motor training-induced plasticity. $\mathrm{J}$ Neuroeng Rehabil. 2009;6:8.

19. Jeffery DT, Norton JA, Roy FD, Gorassini MA. Effects of transcranial direct current stimulation on the excitability of the leg motor cortex. Exp Brain Res. 2007;182:281-7.

20. Parazzini M, Fiocchi S, Liorni I, Rossi E, Cogiamanian F, Vergari $\mathrm{M}$, et al. Modeling the current density generated by transcutaneous spinal direct current stimulation (tsDCS). Clin Neurophysiol. 2014;125:2260-70.

21. Cogiamanian F, Vergari M, Pulecchi F, Marceglia S, Priori A. Effect of spinal transcutaneous direct current stimulation on somatosensory evoked potentials in humans. Clin Neurophysiol. 2008;119:2636-40.

22. Truini A, Romaniello A, Galeotti F, Iannetti GD, Cruccu G. Laser evoked potentials for assessing sensory neuropathy in human patients. Neurosci Lett. 2004;361:25-28.

23. Cogiamanian F, Vergari M, Schiaffi E, Marceglia S, Ardolino G, Barbieri S, et al. Transcutaneous spinal cord direct current stimulation inhibits the lower limb nociceptive flexion reflex in human beings. Pain. 2011;152:370-5.

24. Meyer-Frießem CH, Haag LM, Schmidt-Wilcke T, Magerl W, Pogatzki-Zahn EM, Tegenthoff M, et al. Transcutaneous spinal DC stimulation reduces pain sensitivity in humans. Neurosci Lett. 2015;589:153-8.

25. Perrotta A, Bolla M, Anastasio MG, Serrao M, Sandrini G, Pierelli $\mathrm{F}$, et al. Modulation of temporal summation threshold of the nociceptive withdrawal reflex by transcutaneous spinal direct current stimulation in humans. Clin Neurophysiol. 2016;127:75561.

26. Priori A, Ciocca M, Parazzini M, Vergari M, Ferrucci R. Transcranial cerebellar direct current stimulation and transcutaneous spinal cord direct current stimulation as innovative tools for neuroscientists. J Physiol. 2014;592:3345-69.

27. Ahmed Z, Wieraszko A. Trans-spinal direct current enhances corticospinal output and stimulation-evoked release of glutamate analog, D-2,3- ${ }^{3} \mathrm{H}$-aspartic acid. J Appl Physiol. 2012;112:157692.

28. Bennett M. The LANSS Pain Scale: the Leeds assessment of neuropathic symptoms and signs. Pain. 2001;92:147-57.

29. Bryce TN, Budh CN, Cardenas DD, Dijkers M, Felix ER, Finnerup $\mathrm{NB}$, et al. Pain after spinal cord injury: an evidence-based review for clinical practice and research: report of the National Institute on 
Disability and Rehabilitation Research Spinal Cord Injury Measures meeting. J Spinal Cord Med. 2007;30:421-40.

30. Hallström H, Norrbrink C. Screening tools for neuropathic pain: can they be of use in individuals with spinal cord injury? Pain. 2011;152:772-9.

31. Celik EC, Erhan B, Lakse E. The clinical characteristics of neuropathic pain in patients with spinal cord injury. Spinal Cord. 2012;50:585-9.

32. Beck AT, Steer RA, Brown GK. Beck depres sion inventory-II. Vol. 78. San Antonio, TX: Psychol Corporation; 1996. p. 490-8.

33. Woods AJ, Antal A, Bikson M, Boggio PS, Brunoni AR, Celnik $\mathrm{P}$, et al. A technical guide to tDCS, and related non-invasive brain stimulation tools. Clin Neurophysiol. 2016;127:1031-48.

34. DaSilva AF, Volz MS, Bikson M, Fregni F. Electrode positioning and montage in transcranial direct current stimulation. J Vis Exp. 2011;pii:2744. https://doi.org/10.3791/2744.

35. Jensen MP, Karoly P. Self-report scales and procedures for assessing pain in adults. In: Turk DC, Melzack R, editors. Handbook of pain assessment. New York: Guilford Press; 1992. p. $135-51$.

36. Farrar JT, Young JP Jr, LaMoreaux L, Werth JL, Poole RM. Clinical importance of changes in chronic pain intensity measured on an 11-point numerical pain rating scale. Pain. 2001;94:149-58.
37. Melzack R. The McGill Pain Questionnaire: major properties and scoring methods. Pain. 175;1:277-99.

38. Olsen MF, Bjerre E, Hansen MD, Tendal B, Hilden J, Hróbjartsson A. Minimum clinically important differences in chronic pain vary considerable by baseline pain and methodological factors: systematic review of empirical studies. J Clin Epidemiol. 2018;101:87-106.

39. Salaffi F, Stancati A, Silvestri CA, Ciapetti A, Grassi W. Minimal clinically important changes in chronic musculoskeletal pain intensity measured on a numerical rating scale. Eur J Pain. 2004;8:283-91.

40. Boggio PS, Nunes A, Rigonatti SP, Nitsche MA, Pascual-Leone A, Fregni F. Repeated sessions of noninvasive brain DC stimulation is associated with motor function improvement in stroke patients. Restor Neurol Neurosci. 2007;25:123-9.

41. Ngernyam N, Jensen MP, Arayawichanon P, Auvichayapat N, Tiamkao S, Janjarasjitt $\mathrm{S}$, et al. The effects of transcranial direct current stimulation in patients with neuropathic pain from spinal cord injury. Clin Neurophysiol. 2015;126:382-90.

42. Schweizer LM, Zahn PK, Pogatzki-Zahn EM, Magerl W, Tegenthoff M, Meyer-Frießem CH, et al. Influence of transcutaneous spinal stimulation on human LTP-like pain amplification: a randomized, double-blind study in volunteers. Clin Neurophysiol. 2017;128:1413-20. 